\title{
Real time Intelligent Control Laboratory (RT-ICL) of PowerLabDK for smart grid technology development
}

Ostergaard, Jacob; Wu, Qiuwei; Garcia-Valle, Rodrigo

Published in:

2012 IEEE Workshop on Complexity in Engineering

Link to article, DOI:

10.1109/CompEng.2012.6242948

Publication date:

2012

Document Version

Early version, also known as pre-print

Link back to DTU Orbit

Citation (APA):

Ostergaard, J., Wu, Q., \& Garcia-Valle, R. (2012). Real time Intelligent Control Laboratory (RT-ICL) of PowerLabDK for smart grid technology development. In 2012 IEEE Workshop on Complexity in Engineering: COMPENG 2012 (pp. 61-64). IEEE. https://doi.org/10.1109/CompEng.2012.6242948

\section{General rights}

Copyright and moral rights for the publications made accessible in the public portal are retained by the authors and/or other copyright owners and it is a condition of accessing publications that users recognise and abide by the legal requirements associated with these rights.

- Users may download and print one copy of any publication from the public portal for the purpose of private study or research.

- You may not further distribute the material or use it for any profit-making activity or commercial gain

- You may freely distribute the URL identifying the publication in the public portal 


\title{
Real Time Intelligent Control Laboratory (RT-ICL) of PowerLabDK for Smart Grid Technology Development
}

\author{
Jacob Østergaard, Qiuwei Wu, Rodrigo Garcia-Valle \\ Centre for Electric Technology (CET), Department of Electrical Engineering \\ Technical University of Denmark (DTU) \\ Kgs. Lyngby, 2800 Denmark
}

\begin{abstract}
This paper presents the Intelligent Control Laboratory (ICL) of the PowerLabDK and describes examples of ongoing research work utilizing the ICL. The ICL is comprised of a real time digital simulator (RTDS) with 5 racks, a full scale SCADA system and experimental control room with a link to the Bornholm power system data, an IBM blade server for optimization and control implementation, and a Phasor Measurement Unit (PMU) Lab. It is possible to interface PMUs and other hardware with the RTDS for hardware-in-the-loop (HIL) and power-hardware-in-the-loop (PHIL) tests. The ICL can interface with the Electric Laboratory through a 4-quardrant power amplifier with $150 \mathrm{~kW}$ continuous power supply capability, Omicron and Doble amplifiers, relays, an electric vehicle with vehicle-to-grid (V2G) capability, LabCell boards, photovoltaic (PV) panels, and micro combined heat plant ( $\mu \mathrm{CHP})$ units. The interactive simulation platform with real power system data and distributed energy resources (DER) hardware makes the ICL a very well-suited test platform for smart grid technology development and validation. The ongoing research work with the ICL illustrates the capability and feasibility of using it as a platform for smart grid technology development.
\end{abstract}

Keywords- Intelligent Control Laboratory (ICL), real time digital simulator (RTDS), hardware-in-the-loop (HIL), powerhardware-in-the-loop (PHIL), smart grid

\section{INTRODUCTION}

In order to handle the climate change challenge, three main measures have been developed, i.e. utilization of renewable energy sources (RES) like wind and solar power, electrification of transport and heating sectors, and improvement of energy efficiency. However, it is quite challenging to deal with the intermittency of RES from the power system planning and operation perspectives. A substantial demand increase from electric vehicles (EVs) and electric heating is another major challenge. The promising part of the scheme is that EVs and electric heating demands can be distributed resources for handling the generation fluctuation from RES if the EV and electric heating demands can be managed in a proper way. Therefore, the smart grid concept has been proposed and is being intensively studied over the world.

Literally, smart grids are "Electricity networks that can intelligently integrate the behaviour and actions of all users connected to it - generators, consumers and those that do both - in order to efficiently deliver sustainable, economic and secure electricity supplies" [1].

Denmark is a unique place for renewable energy utilization and smart grid technology development. In 2011 the wind power generation covered $28 \%$ of the demand (incl. grid losses). The Danish parliament has entered a new energy agreement and set a target of $50 \%$ penetration of wind power in 2020 and $100 \%$ penetration of renewable energy in 2035 [2]. In order to handle the challenges faced by transforming the electric power system, ensure Denmark and DTU to have a strong position in smart grid technology development, and make it possible for Denmark to be a global smart grid hub, a world class experimental platform named PowerLabDK, has been established [3].

The PowerLabDK is an international unique experimental platform ranging from basic research to large-scale and fullscale experiments. It is comprised of the High Power Lab in the Copenhagen University College of Engineering (IHK), the Intelligent Control Lab (ICL), Electric Lab, High Voltage Lab and Power Student Lab in DTU Lyngby campus, a large scale test system (SysLab) and the Power Flex House facility in DTU Risø campus and a full scale power system in the island of Bornholm (27,000 customers and 33\% wind power penetration).

The ICL is a simulation platform comprising a real time digital simulator (RTDS), a full scale ABB Network Manager SCADA system, a full-scale experimental power system control room, an IBM blade centre for optimization and control implementation, a PMU lab, a $150 \mathrm{~kW}$ power amplifier, and 21 LabCell boards. Furthermore, a number of small equipments are available in the ICL, e.g. an electric vehicle (EV) with vehicle to grid (V2G) capability, an Omicron CMS 156 voltage and current amplifier, a Siemens 7SJ6416-5EB92-1HH0 multifunction protective relay. The ICL setup makes a very wellsuited platform to carry out proof-of-concept tests using hardware-in-the-loop (HIL) and power-hardware-in-the-loop (PHIL) testing methods.

A HIL test is a technique used to understand the behaviour of a new device, to develop and test complex systems, or to predict an outcome under different system conditions. A HIL simulation often refers to a system in which part of a full 
simulation has been replaced with actual physical components [4], [5]. Based on the coupling type of actual physical components with the simulation part in HIL; HIL can generally be classified into two categories - controller hardware-in-theloop (CHIL) and power hardware-in-the-loop PHIL. A CHIL test is a scenario where there is no power transfer to or from the hardware being tested, in contrast to the more challenging case in a PHIL test, where real power is virtually exchanged between the simulation case and the actual hardware. Appropriate power amplification and conversion apparatuses must be included. The closed-loop PHIL of the device and the network model provides insights into both the performance of the control scheme, and its effect on the real hardware and system performance. Hence, PHIL simulation is an effective method to test or verify the functionality of new control systems and different power components interactions [6], [7]. Therefore, the HIL and PHIL tests are excellent ways of testing and validating smart grid technologies.

The rest of the paper is arranged as follows. In section II, the ICL is described in detail. A few examples of using the ICL for smart grid technology testing are presented in Sections III, IV and V, respectively. In the end, a brief conclusion is drawn.

\section{REAL TIME INTELLIGENT CONTROL LABORATORY}

The ICL is a full scale power system control room for technology development without disturbing real power system operation or real customers. The system setup of the ICL is shown in Figure 1.

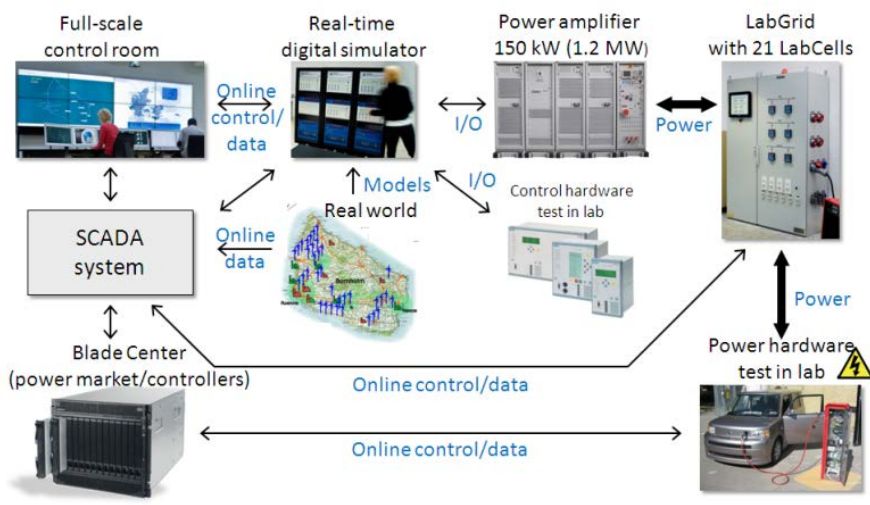

Figure 1 The ICL system setup

The ICL comprises a real time digital simulator (RTDS), a full-scale SCADA system and full-scale experimental power system control room, with a link to the Bornholm power system data, an IBM blade centre for optimization and control implementation, and different hardware interfacing with the RTDS for hardware-in-the-loop (HIL) and power-hardware-inthe-loop (PHIL) tests. The hardware in the Electric Lab integrated with the ICL is an Spitzenberger \& Spies power amplifier with $150 \mathrm{~kW}$ continuous power supply capability, a LabGrid with 21 LabCell boards, Omicron amplifiers, relays, an electric vehicle with vehicle-to-grid (V2G) capability, , photovoltaic (PV) panels, and micro combined heat plant $(\mu \mathrm{CHP})$ units.

The ICL has a RTDS with 5 racks. The RTDS racks are equipped with 4 GPC cards for each and the RTDS system can simulate a power system with up to 110 buses. The RTDS system will be upgraded with PB5 cards for the existing racks and will add 5 more racks. After the upgrade is finished, it will be able to simulate a power system up to 480 buses. It will be a very good simulation platform for both large scale transmission systems and distribution networks with quite large number of buses.

The RTDS, SCADA system and experimental control room can be used for several different applications including:

- Power system operation

- Operator training

- $\quad$ SCADA and EMS applications

- Human-machine interface

- Data collection from field

- Supervision and control of experiments

The combination of the RTDS, SCADA system, the full scale control room, and Bornholm power system data and model creates an interactive environment for simulating real power system operation and control, and a training tool for system operators.

The modular blade server infrastructure is a flexible computer facility which can be accessed locally or remotely. It consists of two IBM blade centres with 36 quad-core CPUs and 856 GB RAM memory. It can be used for optimization and controller implementation purposes. The current applications are:

- Electricity market simulation

- $\quad$ EV fleet management systems

- Virtual power plant development and test

- Power system analysis

- Data collection and processing from field

- Control room applications

The $150 \mathrm{~kW}$ power amplifier is a very powerful three-phase four-quadrant power unit with unique characteristic for test, lab supply and combined grid studies. It can supply ideal and disturbed voltages up to $150 \mathrm{~kW}$ continuous as source and 42 $\mathrm{kW}$ continuous as sink. It has very fast slew rate $(>52 \mathrm{~V} / \mathrm{ms})$ and large signal bandwidth (DC - $5 \mathrm{kHz}$ ). The high peak load ability of the amplifier is $1.2 \mathrm{MW}$. It can be used for different applications:

- $\quad$ EMC component tests (IEC/EN 6100-4-11 etc.)

- Solar power inverters and wind turbines

- High power electronic units (harmonics, voltage dips, loss of phase, transients, phase shifts, ...)

- $\quad$ Power line carriers

- Combined grid studies: realistic grid voltage generated in real-time in RTDS $\rightarrow$ signal amplification in amplifier $\rightarrow$ physical equipment in lab

The LabCell boards distributed in the 3,000 m2 laboratory building at DTU Lyngby campus are the main interface for experiments. There are totally 21 LabCell boards. Each LabCell board is a general purpose main interface which can serve as power interface, SCADA and control interface and communication interface. The LabCell boards are connected in a LabGrid which is similar to a normal power distribution grid and is split into three ring feeders. 


\section{HIL - TEST OF ACTIVE DistRIBUTION NETWORK CONTROLLER}

With the RTDS facility in the ICL lab, a simulation platform has been established with Matrikon OPC server for SCADA DNP 3 and an agent based controller for testing active distribution network controllers [8], [9]. The simulation platform is shown in Figure 2.

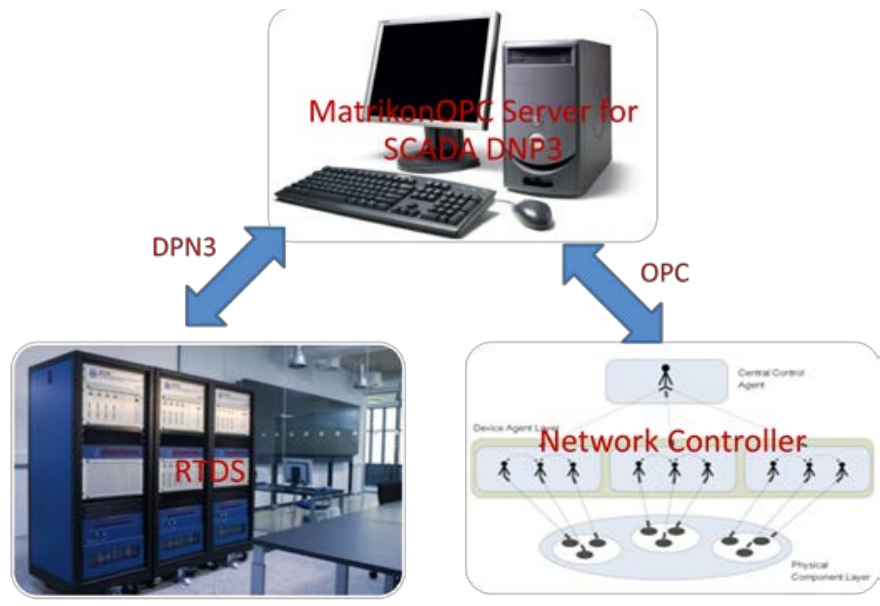

Figure 2 Simulation platform for active distribution network controller

The Matrikon OPC server for SCADA DNP3 is a communication protocol gateway which enables the data exchange between systems using DNP3 protocol and systems using OPC protocol.

In the simulation platform in Figure 2, the RTDS has been used to simulate the Bornholm power system and provide realistic dynamic performance of the simulated power system under both steady state and fault conditions. The simulated power system can interact with external systems using the DNP3 protocol through the GENET card of the RTDS system.

On the right hand side of Figure 2, an agent-based controller developed in the JADE platform is shown as an example. The developed agent based controller is intended to control the distributed generators (DGs) in the Bornholm power system in order to stabilize frequency and voltage after the Bornholm power system enters islanding operation mode. The developed agent-based controller can interact with external systems using the OPC protocol.

With such a system setup, the developed agent-based controller for active distribution networks has been tested and verified with realistic dynamic performance of Bornholm power systems [9].

\section{HIL - TEST OF DIRECTIONAL OVERCURRENT PROTECTION FOR OFFSHORE WIND FARMS}

This section encompasses the establishment of a testing platform, for over-current protection relays of the Walney offshore wind farm [10]. In order to get at fully functional HIL of the power system, the laboratory setup is desired to make a precise simulation of the power system in the RTDS of the Walney offshore wind farm. This simulation model can be used to observe the behaviour of the protection relays that occurs during intolerable operating conditions and compare them with the results from the Walney offshore wind farm. The HIL test can be used to verify the system response as well as to improve the relay settings under unexpected situations [11]. Figure 3 shows a diagram connection with two relays, where amplifiers are used to produce the required voltage and current levels, Ethernet connection is used to establish feedback connection.

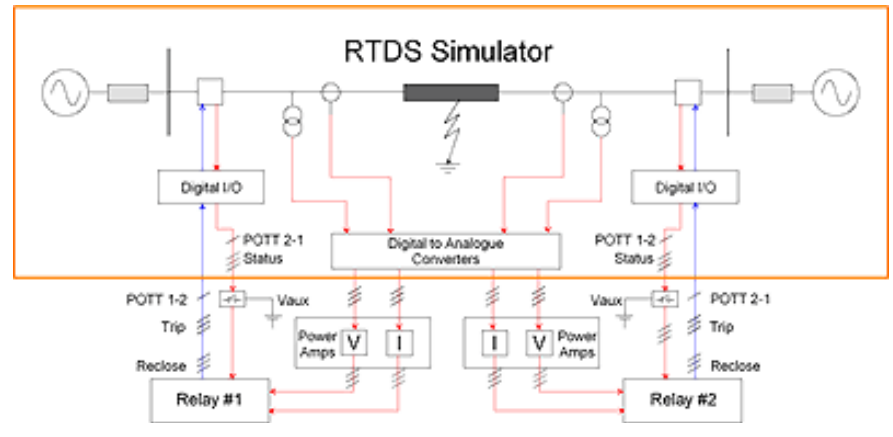

Figure 3 Closed-loop testing of protective relay.

\section{PHIL TEST OF VSC HVDC CONNECTION FOR OFFSHORE WIND POWER PLANTS}

The RTDS and the power amplifier have been used to establish a PHIL test platform for VSC HVDC connection for offshore wind power plants with a VSC to replicate the plant side VSC station of the VSC HVDC connection [12]. The test platform is shown in Figure 4.

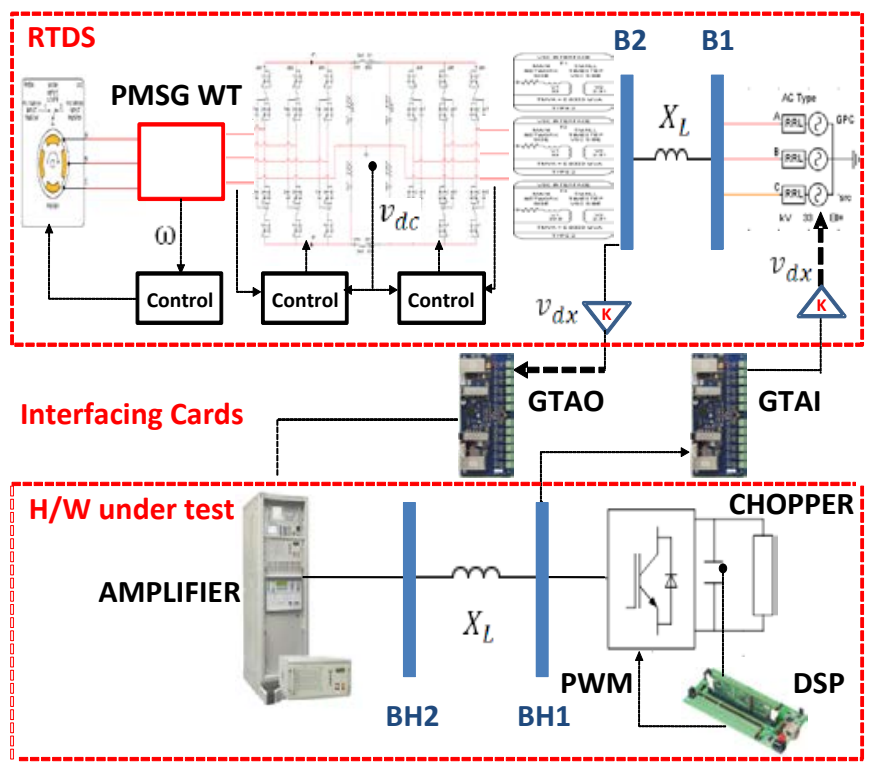

Figure 4 PHIL test platform for VSC HVDC connection for offshore wind power plants

In the PHIL test setup, an offshore wind power plant (WPP) with full scale converter wind turbine generators (FSCWTGs) has been simulated in the RTDS by a scaled up FSCWTG model and a controlled voltage source to represent the plant side VSC. The simulated WPP interacts with external hardware through GTAO and GTAI cards.

The external hardware consists of a S\&P power amplifier, a $\mathrm{VSC}$, a resistive chopper and inductor for connecting the power amplifier and the VSC.

A feed forward DC link voltage control algorithm has been tested for fault handling of the VSC HVDC connection for offshore wind power plants [12]. 


\section{CONCLUSION}

The setup of the ICL of PowerLabDK has been presented in detail. The system architecture, simulation tools and hardware in the ICL create a unique experimental platform to test newly developed technologies of smart grids and support the research and development in the smart grid field. The feasibility of using the ICL to test new technologies of smart grids has been illustrated by test of agent based controller for active distribution networks, HIL test of protection scheme for offshore wind power plants and PHIL test of VSC HVDC for offshore wind power plants.

The ICL will be used to implement tests for the ongoing research in Centre for Electric Technology (CET), Technical University of Denmark (DTU), such as development of widearea systems for secure operation of renewable-based power systems, multi-terminal HVDC for large scale offshore wind power integration, etc.

\section{REFERENCES}

[1] EU Technology Platform for the Electricity of the Future, http://www.smartgrids.eu/

[2] Agreement between the Government and Venstre, Danish People's Party, Red-Green Alliance and Conservative People's Party about the Danish energy policy 2012-2020 (in Danish: Aftale mellem regeringen (Socialdemokraterne, Det Radikale Venstre, Socialistisk Folkeparti) og Venstre, Dansk Folkeparti, Enhedslisten og Det Konservative Folkeparti om den danske energipolitik 2012-2020), March 2012, http://www.kemin.dk/Documents/Presse/2012/Energiaftale/Aftale\%202 2-03-2012\%20FINAL.doc.pdf.

[3] PowerLabDK - Experimental platform for electric power and energy, www.powerlab.dk.
[4] X. Wu, S. Lentijo, and A. Monti, "A novel interface for powerhardware- in-the-loop simulation," in Proc. of IEEE Workshop on Computers in Power Electronics 2004 pp. 178 - 182, Aug. 2004.

[5] S. T. Cha, I. K. Park, Q. Wu, A. H. Nielsen and J. Østergaard, "Realtime hardware-in-the-loop (HIL) testing for power electronics controllers," in Proc. of APPEEC 2012, Mar. 2012.

[6] M. Steurer, F. Bogdan, W. Ren, M. Sloderbeck, and S. Woodruff, "Controller and power hardware-in-loop methods for accelerating renewable energy integration,” in Proc. of PES General Meeting 2007, pp. $1-4$, June 2007.

[7] W. Ren, M. Sloderbeck, M. Steurer, V. Dinavahi, T. Noda, S. Filizadeh, A. Chevrefils, M. Matar, R. Iravani, C. Dufour, J. Belanger, M. Faruque, K. Strunz, and J. Martinez, "Interfacing issues in real-time digital simulators," IEEE Transactions on Power Delivery, Vol. 26, No. 2, pp. 1221 -1230, April 2011.

[8] Q. Wu, S. T. Cha, A. Saleem and J. Østergaard, Communication test for MatrikonOPC server for SCADA DNP 3 with RTDS, CET, DTU Elektro, Internal report, Aug. 2010.

[9] S. T. Cha, Q. Wu, J. Østergaard and A. Saleem, "Multi-agent based Controller for Islanding Operation of Active Distribution Networks with Distributed Generation", in Proc. Of DRPT 2011, pp. 803-810.

[10] http://www.dongenergy.com/walney/Pages/index.aspx Available online.

[11] A. Hadzisakovic, BSc Thesis, "HIL - Testing for Directional OverCurrent Protection for Offshore Wind Farms", Technical University of Denmark, Department of Electrical Engineering, Nov. 2011.

[12] R. Sharma, S. T. Cha, Q. Wu, T. Rasmussen, K. H. Jensen and J. Østergaard, "Power-Hardware-In-the-Loop (PHIL) Test of VSC HVDC connection for Offshore Wind Power Plants", in Proc. of the $10^{\text {th }}$ International Workshop on Large Scale Integration of Wind Power into Power Systems as well as on Transmission Networks for Offshore Wind Power Plants, Aarhus, Denmark, 2011. 